\title{
UN CASO DE DESARROLLO TECNOLOGICO EN CUBA: EL FERROCARRIL
}

\section{Eduardo L. Moyano Bazzani}

\section{Introducción}

Dentro de los notables cambios tecnológicos surgidos a lo largo del siglo XIX, el ferrocarril fue, sin duda, uno de los de mayor trascendencia. Si bien los "caminos de hierro» existían desde fines del siglo XVI en Alemania e Inglaterra, utilizados, entre otras actividades, para el transporte de vagones de mineral desde las bocaminas, su peculiaridad es que éstos eran arrastrados por fuerza animal.

La innovación en los procedimientos de transporte provino de la aplicación del vapor, cuyos ventajosos resultados se evidenciaron en 1829, cuando George Stepheson y su hijo Robert, diseñador y constructor, respectivamente, de la locomotora Rocket, vencieron en las difíciles pruebas de Rainhill (Liverpool), al cubrir su máquina un trayecto de 20 kilómetros en sólo 53 minutos. A partir de entonces, la Rocket fue seleccionada como pieza motriz de los nuevos trenes que empezarían a correr entre Liverpool y Manchester, sustituyendo la locomotora a vapor a la tracción de sangre utilizada hasta entonces en los caminos de hierro (1).

Los Stepheson consiguieron incrementar la potencia y energía de la Rocket mediante la incorporación de elementos tan simples como la caldera tubular y el tiro de vapor de escape del cilindro, que situaron a nivel de la chimenea, imprimiendo a la locomotora una velocidad de 12 millas (19,2 kilómetros) por hora. 
Su éxito se debió, fundamentalmente, a que supieron valorar la conjunción existente entre el vehículo y el carril, relación que habría de resultar trascendental para el desarrollo de la industria y los sistemas de transporte. Los caminos de hierro se convertirían en arterias a través de las cuales fluirían las materias primas y los productos elaborados desde los núcleos productivos a los puntos de difusión.

\section{El ferrocarril en Cuba}

La era del ferrocarril en Cuba comenzó en 1830, año en que en Inglaterra quedó comprobado de manera indiscutible que el ferrocarril era un medio comercialmente muy valioso aplicable al movimiento de carros de tracción animal existentes hasta entonces.

De esa misma fecha data, asimismo, la propuesta de Marcelino Calero Portocarrero (2) para difundir ese avance tecnológico en Cuba, y así, tanto la comunicación oficial de la misma a la Sociedad Económica por el Capitán General de la Isla, Francisco D. Vives, como las Actas de la Sesión de dicha entidad de 22 de julio de 1830 , vendrían a constituir los primeros documentos históricos que se conocen sobre la instalación del ferrocaril en Cuba.

Su primer tramo, de 27,2 kilómetros, tendido entre las localidades de La Habana y Bejucal, se inauguró el 19 de noviembre de 1837, convirtiéndose en el pionero de Las Antillas y del mundo iberoamericano, anticipándose en casi once años al primer ferrocarril peninsular de Barcelona a Mataró.

La primera línea cubana quedó finalizada un año más tarde, cubriendo el área entre la ciudad de La Habana y la rica villa de Güines, con un recorrido total de 71,2 kilómetros que atravesaba el centro de la región productora de azúcar más importante de aquel período.

En efecto, la rápida expansión del tendido ferroviario estuvo en relación directa con el desarrollo productivo del azúcar cubano y su colocación en los mercados mundiales, hasta convertir a Cuba en un núcleo económico de primer orden desde principios del siglo XIX. La exigencia de cubrir mayores distancias y el incremento de los volúmenes de carga a medida que crecían las áreas dedicadas a la producción, pusieron de manifiesto la urgencia de renovar los sistemas de transporte, sustituyendo el ferrocarril al tráfico de carretas por caminos deteriorados y prácticamente intransitables durante el período de lluvias.

El gobierno colonial actuó como promotor nominal de la empresa del 
ferrocarril a través de la administración de la Junta de Fomento, integrada por las más poderosas familias cubanas. El primer proyecto, financiado eventualmente por un préstamo contraído bajo la garantía de España en Londres, no tardaría en mostrarse, al poco tiempo del inicio de su explotación, como un elemento eficaz y beneficioso aplicable a las necesidades cubanas. La comprobación de las posibilidades económicas y materiales de su construcción y la rentabilidad obtenida, impulsaron nuevos proyectos que, esta vez financiados con capitales cubanos, llegarían a establecer, en treinta y cinco años, una red de líneas férreas regionales concentradas, principalmente, en la parte occidental de la Isla.

El gobierno de Madrid concedió importantes privilegios, entre ellos garantías perpetuas sobre concesiones durante largos períodos, una ley sobre expropiación compulsatoria de tierras y la exención de gravámenes para todo el material y equipo pesado utilizado en las obras.

Hasta finales de la década de 1850, la extensión de los trabajos específicos del ferrocarril fue rápida. Los principales puertos de la Isla, La Habana, Matanzas, Cárdenas, Caibarién, Guantánamo y Cienfuegos, quedaron unidos con el interior por medio de líneas férreas, cumpliéndose así uno de los objetivos centrales de los hacendados: abrir vías hacia los puertos para facilitar la exportación de los diferentes productos.

Sin embargo, la mayoría de las empresas ferroviarias se vieron frecuentemente al borde de la quiebra, como en los años 1852-1854 cuando, al no lograr reunir montos de capital suficiente, se vieron incapacitadas para cumplir con los compromisos contraídos. Pero estas dificultades no obstaron para que el espíritu de asociación para emprender este tipo de obras, desarrollado desde el tendido de las primeras líneas, se hiciera progresivamente más intenso, impulsado en gran parte por las disposiciones del gobierno al conceder ciertas garantías a los capitales que se aplicaran a esta industria.

El momento clave en la extensión de líneas férreas fue la década de 1850-1860, período durante el cual no hubo un solo año en que no se pidieran autorizaciones para acometer proyectos ferroviarios. Estos fueron destinados a las principales zonas productoras del país, especialmente áreas azucareras. Es significativo en este sentido el caso de la jurisdicción de Matanzas, que comprendía los términos de Cárdenas y Colón, donde, basándose en los resultados de la línea Habana-Güines, proliferaron los proyectos y el tendido de líneas férreas, generándose, posteriormente, una gran competencia y rivalidad entre las diferentes Compañías.

La disposición del tendido de las líneas siguió un modelo radial, uniendo las zonas interiores con los puertos más cercanos, dando origen a una 
red totalizadora que integraba ferrocarriles locales o líneas intermedias, como las de la zona central y oriental, con los grandes ramales principales.

Las líneas secundarias fueron de construcción más tardía, dado que, al atravesar zonas poco desarrolladas o improductivas, los costos resultaban elevados, disminuyendo las perspectivas de beneficio para las empresas.

Ya a finales de la década de 1860, el tendido ferroviario no se desarrollaría con la misma magnitud, si bien se promovieron concesiones de nuevas líneas y se procedió a concluir o perfeccionar las construidas hasta el momento.

Si el cálculo de lo realizado en 1850 se cifró en 535 kilómetros, el tendido había alcanzado, ocho años después, una extensión de 1.302,054 kilómetros planificados, financiados y ejecutados por capitales extranjeros. A finales de 1868, el capital invertido en ferrocarriles sumaba un total de 38.701 .630 pesos (3).

Una mirada al mapa de los ferrocarriles en 1868 muestra claramente que la mayor parte de las líneas cubrían las regiones del sur y este de La Habana, contando el resto de la Isla sólo con algunas conexiones parciales. El ferrocarril central, que uniendo el este con el oeste podría haber facilitado la integración del país, no fue construido en su totalidad hasta fines de siglo.

Otro elemento digno de resaltar fue la mano de obra utilizada en la instalación del ferrocarril cubano (4). Siendo de dos tipos, especializada y no profesional, contó la primera con directores y técnicos, ingenieros, dibujantes y ayudantes de nacionalidad norteamericana, inglesa y francesa y la segunda con artesanos y jornaleros cubanos, prisioneros de guerra enviados por el gobierno peninsular, peones irlandeses y canarios contratados expresamente para la obra, emancipados africanos retenidos ilegalmente en servidumbre, esclavos propiedad de la Junta de Fomento o alquilados por sus amos, cimarrones y, sólo más tarde, culíes.

$\mathrm{Si}$ al primer grupo correspondieron las responsabilidades de la toma de decisiones y la resolución de los problemas técnicos y constructivos, recayó en el segundo el peso efectivo de las penosas tareas del tendido ferroviario y las condiciones de trabajo infrahumanas a que se vieron sometidos.

Así pues, la adopción en Cuba del progreso tecnológico aplicado al transporte convivió y propició renovados sistemas de explotación de la fuerza de trabajo. 


\section{Las primeras locomotoras en Cuba}

La temprana aparición del ferrocarril en Cuba tiene un significado que trasciende el mero hecho de su prioridad mundial o regional, al ejemplificar la forma en que se decidió el control del mercado ferroviario que se abría en la Isla con la introducción de las locomotoras.

Inglaterra, pionera del ferrocarril moderno y erigida en dueña absoluta del mercado ferroviario internacional hasta la década del 30 del siglo pasado, fue el núcleo proveedor de tecnología y equipamiento pesado al resto de los países, papel exportador del que no estuvo excluido Estados Unidos. Si en los primeros momentos el mercado norteamericano se nutrió de la tecnología inglesa, posteriormente ambos países adquirieron caracteres diferenciados que permitieron abrir nuevas brechas en el horizonte científico-técnico ferroviario.

Las locomotoras norteamericanas, dotadas de peculiaridades propias, estuvieron en disposición de ofrecer al mercado mayor ligereza, flexibilidad operativa y ahorro. Estas máquinas de vapor asimilaban leña como combustible, tenían seis u ocho ruedas e incluían un truck delantero giratorio que les imprimía mayor seguridad en las curvas; asimismo, el espantavacas o rejilla delantera para apartar obstáculos de la vía las distinguía. Incorporaron con rapidez variantes, tanto en la disposición de los cilindros como en la composición de los metales de la caldera y tubos, entre otras innovaciones (5).

Las primeras máquinas que llegaron a Cuba fueron comercializadas por el londinense Alejandro Robertson, comerciante que actuó también como intermediario en la negociación del empréstito para la construcción del ferrocarril. Robertson se constituyó en el agente de la Junta de Fomento cubana en Inglaterra para la adquisición de raíles, locomotoras, combustible, herramientas, carros y coches. El modelo de locomotora elegido fue el Rocket de George Stepheson.

Por medio de Robertson, se compraron ocho máquinas de vapor de un tipo similar a las utilizadas en la línea Londres-Birmingham a un precio de 64.000 pesos, y se encargó la construcción de cuatro locomotoras de pasajeros y otras cuatro para mercancías a los fabricantes John Braithwaites y Reanis, las cuales, recibidas a principios de 1837, surcaron por primera vez las tierras cubanas el 19 de noviembre de 1838.

El peso de los vehículos oscilaba entre las diez y doce toneladas; poseían cuatro grandes ruedas rígidas, todas de igual diámetro y capacidad para arrastrar algo más de 25 toneladas en una pendiente máxima de 45 pies por milla. Entre otras particularidades, estos equipos poseían cilindros de 
doce pulgadas de diámetro diseñados para quemar carbón mineral y lograr una presión de sesenta libras por pulgada cuadrada (6).

Los primeros meses de explotación no dieron, sin embargo, los resultados esperados. La tónica estuvo marcada por la alteración de los itinerarios establecidos, a causa de descarrilamientos, rotura de máquinas, accidentes provocados por animales y hasta choques de locomotoras, situación que se agravó a mediados de 1839, llegándose a paralizar el servicio. A partir de entonces, se manifestaron continuas controversias entre los ingenieros, técnicos y maquinistas ingleses que operaban con las locomotoras, y el ingeniero director de obras, el norteamericano Alejandro Cruger. El diseño de la vía del ferrocarril habanero, realizado por ingenieros norteamericanos, fue criticado por los especialistas ingleses, quienes lo consideraban inadecuado, achacando los accidentes ocurridos a los defectos de su trazado.

Cruger, por su parte, planteó la necesidad de incorporar dos locomotoras del modelo norteamericano y de contratar maquinistas experimentados de ese país. Luego de visitar varias fábricas, optó finalmente por el establecimiento Baldwin, donde adquirió dos máquinas de vapor por 14.500 pesos, una de ellas para el transporte de carga y la otra, más ligera, para pasajeros, ambas con seis ruedas y truck delantero. El cambio de la posición de las ruedas motrices hacia la parte posterior de la caldera, la difusión del bogie, su particular disposición y corta distancia entre sus ejes, permitía que corrieran sin dificultad por curvas de menor radio que las europeas, sin estar expuestas a salirse de las vías, innovaciones que las capacitaban para arrastrar convoyes de 150 toneladas y penetrar en zonas no accesibles hasta entonces (7).

Estas máquinas, las primeras exportadas desde Estados Unidos en 1838, eran muy superiores técnicamente a las inglesas, se alimentaban con leña y gastaban más de una cuerda en cada viaje a Güines. Su llegada a Cuba fue acompañada de un equipo de técnicos norteamericanos contratados por Cruger que no tardaron en sustituir a los ingleses.

Los resultados de estas nuevas máquinas Baldwin fueron excelentes, restableciéndose así la confianza en el ferrocarril, cuyos funcionarios retiraron de la circulación las locomotoras inglesas e iniciaron los trámites para la devolución a Gran Bretaña de todo el material y la exigencia de reclamaciones.

A fines de 1838 arribaron a La Habana dos nuevas locomotoras adquiridas en Estados Unidos, una fabricada por Norris y otra por Baldwin; de esta forma, el servicio ferroviario completado hasta Güines quedó cubierto únicamente con equipos norteamericanos. 
Los coches para pasajeros, de la misma procedencia, presentaban las siguientes características: los de primera clase tenían ventanillas corredizas, el techo cubierto con cuero fuerte, molduras y manijas de cobre. El exterior era de caoba muy perfeccionada en su línea. En los asientos había cojines de paño. En cuanto a los de segunda clase, la descripción contemporánea asegura que eran parecidos a los de primera; las dimensiones de ambos eran de cinco varas de fondo por dos varas y siete pulgadas de anchura, y una vara y tres cuartos de alto del techo. Los coches de tercera tenían cuatro varas y media de largo por dos y media de ancho, siendo invariable la altura respecto a los anteriores, no así la madera utilizada en cada uno de ellos (8).

Una nota pintoresca de Samuel Hazart comenta:

«...cuando uno entra en los carros del tren, le admira su apariencia seca y aireada. Las ventanillas no tienen cristales, sino simplemente persianas de tablillas muy delgadas para permitir el paso de la mayor cantidad de aire.

Los asientos, así como sus respaldos, son de rejilla.

La manera como dan salida al tren es ridículamente curiosa: no es con los vivos y rápidos sones de una gran campana y el perentorio "señores viajeros al tren", sino por medio de un chino medio cubanizado con blusa azul y zapatillas que camina arriba y abajo del andén sonando una campanilla como si estuviera vendiendo baratijas, y no anunciando la salida del tren...» (9).

Los paraderos y estaciones, así como los edificios destinados al material fijo y móvil, eran en general de reducidas dimensiones, pero tendieron a mejorarse con el tiempo.

En el mes de julio de 1839, apenas un año después de la incorporación del nuevo material norteamericano, la empresa cubana contaba con unos 120 carros de carga y dos coches para pasajeros, al parecer éstos muy cómodos y elegantes. Sobre su operatividad, se calculó que, en caso de ser necesario trasladar tropas, cada máquina podría llevar veinte carros con una veintena de hombres y su equipo correspondiente, estimándose el tiempo para llegar a Güines desde la capital en menos de tres horas.

En cuanto a la velocidad desarrollada, aunque la media oscilaba entre las 15 y 18 millas por hora, llegaron a alcanzarse las 35 o 40 millas en el mismo tiempo (10). 


\section{Sistema y superestructura del ferrocarril. Consideraciones generales}

Haremos alusión en este apartado a aquellos elementos como sistema, terraplenes, alcantarillas de desagüe y puentes carril que, experimentados por primera vez en la línea Habana-Güines, se aplicarían posteriormente en la mayoría de las líneas férreas cubanas hasta fines de 1878 .

El sistema adoptado inicialmente fue el de carriles salientes sobre durmientes longitudinales y traviesas de maderas duras del país o importadas de Estados Unidos, principalmente ciprés, cedro blanco o roble. Pero la escasez de madera obligó a renunciar a los durmientes y reemplazar los travesaños por polines y bancos de sillería; más adelante, advirtiendo los inconvenientes derivados de que se rompieran cuando los trenes llevaban mucha velocidad, o de que crearan desniveles en los carriles, se adosarían travesaños de madera sobre los mismos bancos.

Las traviesas se colocaban a una distancia de dos y medio a tres pies de centro a centro y bien adaptadas a las condiciones del terreno, sujeto a continuas erosiones en los terraplenes por las abundantes lluvias. Sin embargo, a pesar de estas preocupaciones, la tendencia de las empresas a abaratar los costos empleando maderas flojas del norte, obligaría a las compañías a afrontar reparaciones frecuentes y costosas (11).

Para el relleno de los bajos de la vía se empleó el sistema de terraplenes artificiales sin muros ni estacas de contención, dándoles un talud de uno y medio por uno. Este sistema, empleado comúnmente en los Estados Unidos, tuvo muy buen resultado en la Isla, a pesar de que en razón del clima y de la intensidad de los aguaceros se temió que se viesen destruidos con rapidez. Se construyeron terraplenes de hasta 60 pies de altura, utilizando más de 150.000 varas de tierra; soportaban diariamente el paso de seis trenes cargados, pero no bajaron ni media pulgada en tiempo de secas. Los taludes permitieron que al poco tiempo se cubrieran las escarpas de yerma, formando un compacto tejido que neutralizaba los efectos perjudiciales de las lluvias (12).

Las alcantarillas de desagüe construidas en toda la extensión de la línea, eran de sillería labrada con tirantes de madera quiebra-hacha de tercia para el paso de los carriles (13).

En cuanto a las obras de ingeniería, es destacable el tendido de un puente sobre el río Almendares y en el Paraje en difíciles condiciones. Los 12 pies de agua corriente y 25 de fango hicieron preciso establecer la mampostería sobre pilotaje; aun así, por haber quedado alguna cabeza de los pilotes bajo el agua, fue necesario hacer un encajonado que se desaguaba con bombas para poder trabajar en seco. Todo el puente, de 
piedra labrada sobre cuatro pilastras de corona, se elevaba a 30 pies sobre la superficie del agua (14).

Asimismo, la creación de un túnel a poco más de un cuarto de legua del puente, en dirección a Güines, llevó a trazar un socavón del que se extrajeron 150.000 varas cúbicas de tierra, utilizadas parcialmente para hacer un terraplén inmediato. Este paso subterráneo tenía 325 pies de largo, 14 de ancho y 16 de alto. Solamente se construyeron arcos de apoyo en las dos bocas de entrada, a pesar de lo cual no se originó ningún derrumbe, siendo esta obra, junto con la del puente, la más notable de toda la línea Habana-Güines (15).

Las barras-carril solían tener por lo común de 56 a 71 libras de peso por yarda lineal y su longitud variaba de 16 a 18, 20, 22 y 24 pies, dimensiones que se empleaban en las curvas y desviaderos. La llamada $\mathrm{T}$ americana servía para sujetarlas a cada traviesa mediante dos alcayatas cuyas cabezas abrazaban sus rebordes inferiores. El sistema adoptado para unirlas entre sí fue variable, unas veces se utilizaron sillerías con alcayatas en todas las traviesas y otras sólo en las que correspondían a dichas uniones, siendo considerado comúnmente como el mejor el de barra de conexión enlazada por cuatro tornillos alternándolas en cada lado. Otro procedimiento fueron las sillas de plancha de hierro de 31 pulgadas de largo y tres octavos de espesor, las cuales, por medio de unas orejas, abrazaban las barras en sus rebordes inferiores, apoyándose en sus extremos sobre las dos traviesas más próximas, a las cuảes se unían con dos clavos.

Sobre dichas planchas se apoyaban los carriles sujetos por mordazas mixtas de planchuelas de hierro de 16 pulgadas de largo, colocadas por la cara interior de los carriles y trozos de madera de roble de 7 pies y 5 pulgadas exteriormente; se unían a las barras con cuatro pernos de rosca y tuerca, de los que dos abrazaban los trozos de madera, los carriles y la planchuela interior, y los otros dos las dos primeras. Los carriles se colocaban dejando intervalos de un cuarto de pulgada, y para permitir la dilatación se daba a los agujeros por donde pasaban los pernos una forma oblonga en sentido longitudinal.

Todo el sistema se unía a las traviesas con cuatro clavos de 9 pulgadas que aseguraban el trozo de roble que servía de mordaza (16).

El uso del balasto se impuso a principios de la década del 60; hasta ese momento no se había generalizado aún la práctica de utilizar en traviesas o puentes las durísimas maderas cubanas, pues a pesar de que abundaban en distintas regiones del país, las dificultades en la tala y transporte las convertían en un material costoso para la construcción de ferrocarriles. 
En sustitución, se adquiría pino blanco y roble a través de los comerciantes norteamericanos, que abastecían a los puertos cubanos a precios asequibles. Pero las dificultades de conservación por la humedad y el calor haría que las empresas ferroviarias dirigieran su atención a las maderas locales, principalmente quiebra hacha, variedades del júcaro y el jiqui (17).

Con respecto a la tipología viaria, los ferrocarriles cubanos manifiestan, en una primera fase, una marcada uniformidad, influyendo en este hecho que los ingenieros y técnicos norteamericanos continuaron en su mayoría trabajando sucesivamente en distintas empresas a las que aplicaron idénticos criterios tecnológicos y un mismo esquema vial.

La mayor parte de los caminos fueron de una sola vía, detectándose la doble sólo en casos aislados.

Durante los primeros veinte años del tendido de la vía férrea, las paralelas fueron extendidas casi siempre sobre la llamada cama del camino, terraplén de 20 a 21 pies de ancho que se hacía con la tierra y las piedras sobrantes de las mismas excavaciones originadas por los trabajos de construcción. En Cuba, la gran mayoría de las Compañías adoptaron un ancho estándar para sus vías, 1,45 metros, aunque ciertas líneas, en casos excepcionales, instalaron paralelas con un ancho menor o medidas superiores, 1,60 metros entre ejes, característica incorporada posteriormente a la red ferroviaria española.

\section{Nuevos avances tecnológicos}

Al comenzar la Guerra de los Diez Años (1868), había en Cuba 176 locomotoras y 179 tenders o carros auxiliares para el combustible (18).

Durante los primeros años, la mayor parte del combustible utilizado para los ferrocarriles fue la leña, y sólo en pequeñas cantidades el carbón de piedra exportado desde Norteamérica y Gran Bretaña, pero a fines de 1868, el carbón, fuente de energía más económiea, llegaría a utilizarse en todas las empresas.

Ese mismo año, el total de vagones y coches de pasajeros ascendía a 2.295, de los cuales, 264 correspondían a pasajeros y 2.031 a carga (19).

A partir de 1878, los ferrocarriles en Cuba inician un proceso de cambio tecnológico, principalmente en el mejoramiento del camino y en el aumento de la capacidad de los equipos de tracción. En el primer caso, el cambio consistió en el reemplazo de los carriles de hierro característicos hasta entonces del ferrocarril por los nuevos raíles de superficie de acero, de mucha mayor resistencia al desgaste. En el segundo caso, las locomo- 
toras, se efectuaron una serie de modificaciones que en su conjunto permitieron la construcción de equipos mucho más pesados y veloces que lograban una mayor capacidad de tracción que hizo necesario el reforzamiento de los puentes y, en algunos casos, su completa reconstrucción adosando vigas de hierro.

Entre otros elementos surgidos en la nueva tecnología del ferrocarril, es destacable la aplicación de los frenos al vacío en los trenes de viajeros y más tarde en los de carga, así como la instalación de los cambia-vía automáticos.

Hasta principios de 1870, la regla tecnológica seguida en Cuba en la construcción de vías férreas había sido el empleo de carriles de hierro con un peso que generalmente no sobrepasaba las 60 libras por metro, los cuales se apoyaban sobre la cama de un camino no siempre balastado. El comienzo del uso de aceros tuvo lugar aproximadamente a partir de la década del 70, cuando algunas Compañías, como la del Ferrocarril de la Bahía, decidieron contratar para su funcionamiento ese nuevo tipo de raíles, esperando que duraran cinco o seis veces más que los utilizados hasta entonces (20), aunque indudablemente el costo de los carriles era mayor.

La competencia existente entre las empresas había obligado a buscar un incremento en la capacidad de arrastre de sus equipos, añadiendo varios carros más en los convoyes para favorecer su capacidad operativa. La compra de locomotoras más potentes, que respondieran a las necesidades crecientes del servicio, fue unida a la indispensable adquisición de equipos complementarios como planchas y fragatas para azúcar y jaulas para caña.

Desde un punto de vista general, la incorporación de nuevas técnicas por los ferrocarriles cubanos al finalizar el siglo XIX se había llevado a cabo en la región occidental, fundamentalmente, donde la industria azucarera no había sufrido las consecuencias de la guerra. Esta adaptación a las transformaciones surgió como reacción a la amenazadora competencia y ante la necesidad de mantener y aumentar la rentabilidad del ferrocarril frente a la situación de crisis económica que, al final del siglo, cambió la estructura de propiedad de un gran número de Compañías.

\section{Conclusiones}

Durante buena parte del siglo XIX, Cuba disfrutó de una brillante situación económica a la que contribuyó decisivamente el desarrollo de 
los ferrocarriles. Este avance tecnológico revolucionario, de gran trascendencia en el ámbito mundial, ejerció en Cuba una influencia innegable en el ámbito económico-social que haría de la Isla un territorio de vanguardia en la adopción de innovaciones y uno de los líderes de la Era de los Ferrocarriles,

La instalación y desarrollo de las vías férreas respondió a la acuciante necesidad de proporcionar un medio de transporte cómodo, rápido, seguro y económico para los principales productos agrícolas de la isla que, desde el aislado interior, debían conducirse a los puertos de embarque para su exportación. El ferrocarril aumentó y mejoró la eficacia de las operaciones mercantiles.

Como señala Moreno Fraginals:

«...el ferrocarril y no la máquina de vapor aplicada al trapiche, es el principal elemento de la revolución industrial que trastorna completamente las condiciones cubanas de producción...» (21).

Desde un principio, Cuba adoptó la tecnología más novedosa, primero inglesa y después norteamericana, aplicada al ámbito industrial ferrocarrilero, tratando, asimismo, de conseguir los mejores técnicos, constructores e ingenieros de este ramo, acomodando las adquisiciones a sus posibilidades económicas.

Estas razones explicarían por sí solas el auge inversor volcado en los ferrocarriles desde los núcleos privilegiados habaneros y provinciales tanto como la localización de los caminos de hierro en el territorio isleño. Tan importante ayuda al sector productivo y exportador permitió la continuación de su marcha ascendente.

El tendido ferroviario funcionó, asimismo, como difusor de innovaciones tecnológicas aplicadas a la exportación agrícola. Al tratarse de un transporte sencillo y masivo, se utilizó para el traslado de equipos industriales pesados, delicados y poco manejables, que produjeron un incremento en la capacidad productiva de la plantación y, en consecuencia, una progresiva mecanización de las operaciones agrícolas. 


\section{NOTAS}

Sigla utilizada: A.H.M.M. Archivo Histórico Militar (Madrid).

(1) DaY, John (1976): Trenes, Bruguera, Barcelona.

(2) Marcelino Calero y Portocarrero, fue un escritor, periodista, liberal, agrario y economista; obtuvo del gobierno español el real privilegio de construir en España un Camino de Hierro en Jerez, proyecto que no se concretó. También propuso la construcción de otro en la Isla de Cuba, que finalmente no se llevó a cabo.

(3) Para mayor referencia ver: Moyano Bazzani, Eduardo L. (1986): El Ferrocarril en Cuba 1836-1878. Un elemento de desarrollo económico, social y espacial. Tesis Doctoral, Facultad de Geografía e Historia. Universidad Complutense, Madrid.

(4) Moyano Bazzani, Eduardo L. (1990): «Los recursos humanos en la construcción del primer ferrocarril cubano", en Revista Arbor, N.o 536-537, Madrid, C.S.I.C., pp. 189-202.

(5) WhITE, John A.: «Introduction of the locomotive safety truck». En E.U. National Museum Contribution from the Museum of History.

(6) Hamilton Ellis, C. (1966): Railway History, Studio Vista, Londres. También Robisns, Michael (1965): The Railway Age in Britain and its Impact on the World, Penguiem Brooks, Baltimore. Co.

(7) History of the Baldwin Locomotive Works 1831-1920. (1923). Philadelphia, The Bongham

(8) A.H.M.M. Ultramar, Madrid. Legajo 4-1-2-11.

(9) HazARD, Samuel (1928): Cuba a pluma y lápiz. La siempre fiel Isla de Cuba, Tradc. Adrián del Valle, La Habana, Tomo II, p. 62.

(10) Vide [8].

(11) Anales de la Isla de Cuba, Diccionario administrativo, económico, estadístico y legislativo. (1861), por Félix Erenchum, Año 1856, Madrid, p. 1793

(12) A.H.M.M. Ultramar, Madrid. Legajo 4-1-2-11.

(13) Idem.

(14) Idem.

(15) Idem.

(16) Memoria de Obras Públicas de la Isla de Cuba (1861), La Habana, p. 94-95.

(17) A.H.M.M., Ultramar, Madrid. Legajo 4-1-2-11.

(18) Jaudenes, Antonio María: Memoria de las Obras Públicas de la Isla de Cuba en los años económicos de 1865-66 a 1872-73, La Habana, p. 631.

(19) JAUDENES, Antonio María: Memoria... op. cit., p. 631.

(20) Actas de la Junta Directiva del Ferrocarril de Bahía, Tomo III, p. 132-134. Ver también, A.H.M.M. Ultramar, Madrid. Legajo 4-1-2-11.

(21) Moreno Fraginals, Manuel (1978): El Ingenio. Complejo económico-social cubano del azúcar, La Habana, Edit. Ciencias Sociales, Tomo I, p. 151. 